\title{
Diffuse Postoperative Peritonitis - Value of Diagnostic Parameters AND IMPACT OF EARLy Indication FOR RELAPAROTOMY
}

\author{
F. G. Bader ${ }^{1,2}$, M. Schröder ${ }^{1}$, P. Kujath ${ }^{1}$, E. Muhl1,3, H.-P. Bruch ${ }^{1}$, C. Eckmann ${ }^{1}$ \\ ${ }^{1}$ Department of Surgery, University of Schleswig-Holstein, Campus Lübeck, Lübeck, Germany \\ ${ }^{2}$ Karolinska Institutet, Karolinska Biomics Center (KBC), Stockholm, Sweden \\ ${ }^{3}$ Department of Surgery, Intensive Care Unit, University of Schleswig-Holstein, Campus Lübeck, Lübeck, Germany
}

\begin{abstract}
Objective: Current criteria for performing relaparotomy for suspected peritonitis are non explicit and based on non-quantitative, subjective arguments or hospital practice. The aim of this study was to determine the value of routinely used clinical and diagnostic parameters in early detection of postoperative, diffuse peritonitis (PP). Furthermore, the prognosis and outcome after early indication for relaparotomy in patients with PP compared to community-aquired peritonitis (CAP) was evaluated.

Methods: Between 1999 and 2008, a total of 251 patients with diffuse secondary peritonitis either postoperative (PP) or community acquired (CAP) were analyzed retrospectively. PP $(\mathrm{n}=114)$ and CAP $(\mathrm{n}=137)$ were compared regarding physical examination, MPIScore, APACHE II-Score, evidence of organ failure, laboratory parameters, diagnostic instruments and clinical course. The treatment regimen comprised surgical source control (with/without programmed lavage), abdominal closure and relaparotomy on demand, broad spectrum antibiotic therapy and intensive care support.

Results: The APACHE II-Score (20 CAP vs. 22 PP, p $=0.012$ ), MPI-Score (27 CAP vs. $30 \mathrm{PP}, \mathrm{p}=0.001$ ) and the number of lavages differed significantly. Positive phyiscal testing and signs of sepsis [abdominal pain $(81.6 \%$ PP vs. CAP $97.1 \%, \mathrm{p}=0.03)$, rebound tenderness $(21.9 \%$ vs. $35.8 \%, p=0.02)$, fever $(35.1 \%$ vs. $51.8 \%, \mathrm{p}=0.03)$ ] occurred significantly less often in the PP patients than in the CAP group. Conventional radiography $(66.2 \%)$ and ultrasonography $(44.3 \%)$ had a lower diagnostic sensitivity than did abdominal CT-scan (97.2\%). Mortality was higher in the PP group but did not differ significantly between the two groups (47.4\% PP vs. $35.8 \%$ CAP, $\mathrm{p}=0.06)$.

Conclusion: The value of physical tests and laboratory parameters in diagnosing abdominal sepsis is limited. CT-scanning revealed the highest diagnostic accuracy. A treatment regimen of early relaprotomy appears to be the most reasonable strategy for as early discovery of postoperative peritonitis as possible.
\end{abstract}

Key words: peritonitis - abdominal sepsis - relaparotomy - diagnosis - treatment

\section{INTRODUCTION}

Secondary peritonitis accounts for approximately $90 \%$ of all peritonitis cases in western countries [1]. Within this group diffuse postoperative peritonitis (PP) and abdominal sepsis are common concerns following surgical interventions. The current literature indicates a rate of between 30 and $42 \%$ for diffuse postoperative peritonitis within the subgroup of secondary peritonitis [2-4]. Despite the development of antibiotics and significant improvement in intensive care support, morbidity is high and mortality rates remain between 30-66\% [5-10].

The surgical treatment of PP is primarily aimed at defining source control, followed by debridement of fibrin bedding and abdominal lavage of contaminants and infectious fluids. Nevertheless, the prognosis and outcome of patients with PP is directly related to early diagnosis and stringent treatment interventions. Recently, encouraging data have been published favoring a relaparotomy-on-demand strategy [11]. Current criteria for performing relaparotomy are non-explicit and are based on non-quantitative, subjective arguments or hospital doctrines. Furthermore, it is known that failure of initial antibiotic therapy in patients with complicated intraabdominal infections is associated with higher mortality rates [12]. Multiple scoring systems predicting the development of severe, life-threatening abdominal sepsis have been established but frequently fail to prognosticate the early onset of peritonitis and therefore miss the ideal time point for intervention. Reliable clinical parameters as well as precise diagnostic predictors which allow for precise detection of PP would thus be of paramount importance.

The aim of this retrospective study was to clarify the value of routinely used clinical and diagnostic parameters in early detection of $\mathrm{PP}$ compared to community acquired peritonitis (CAP). Furthermore, the prognosis and outcome after early indication for relaparotomy in patients with PP was evaluated.

\section{Material And Methods}

A total of 251 patients with diffuse secondary peritonitis treated between May 1999 and April 2008 at 
the Department of Surgery, University of SchleswigHolstein Campus Lübeck, were evaluated retrospectively. All consecutive cases within this time-frame were included. This group of patients comprised 114 individuals with PP and 137 patients with CAP, the latter serving as a control group.

All data regarding short and long term medical history, physical examination, MPI-score, APACHE IIscore, laboratory values, imaging procedures (ultrasound, CT-scan, contrast-media imaging, mesenteric angiography), intraoperative findings, operative procedures and postoperative course were collected in our 'peritonitis-database' annually.

Diffuse secondary peritonitis was defined as intraoperative evidence of inflammation of the peritoneal surface and/or contaminants/infectious peritoneal fluid in all quadrants due to an intestinal perforation. CAP and PP were defined accordingly. In addition, $\mathrm{PP}$ was defined as a direct (e.g. anastomotic leakage) or indirect (e.g. perforated gastric ulcer after hemicolectomy) complication of a previously performed abdominal surgery. All cases of localized peritonitis were excluded. Furthermore, patients with suspected peritonitis but negative findings at the time of laparotomy were not included in the peritonitis database.

Diagnostic procedures in the case of suspected secondary diffuse peritonitis included abdominal ultrasound, CT-scan and radiological imaging using contrast-media as well as mesenteric angiography (when appropriate). Indication for relaparotomy after positive findings in CT-scan were based on the following citeria: Evidence of leakage, intraabdomnal air after more than five days postoperatively, and/or massive collection of intraabdominal fluid. The diagnostic significance was defined as 'correct' when correlating with intraoperative findings.

The APACHE II-score, the values for C-reactive protein (CRP), white blood cells (WBC), lactate and antithrombine III (AT III) were all documented daily for the first 7 days and twice a week thereafter until the patients were discharged.
The surgical treatment was primarily aimed at definitive source control, followed by gentle debridement of fibrin bedding and abdominal lavage with lactated Ringer`s-solution and Polyhexanide. Relaparotomy was performed immediately following positive radiological examination and/or indicative clinical/ laboratory signs. In PP, negative radiological findings and persistent symptoms of sepsis for longer than 24 hours were also indications for relaparotomy.

After the intraoperative diagnosis of secondary peritonitis, relaparotomy was performed every 24-48 hours on a scheduled basis (at least once), until intraoperative findings allowed for an abdominal closure. In case of dehiscent abdominal fascia, a Dexon ${ }^{\circledR}$ mesh was used.

\section{STATISTICAL ANALYSIS}

To determine differences between the two groups (PP versus CAP), the $\chi^{2}$-test and Mann-Whitney U-test were used when appropriate. $\mathrm{P}<0.05$ was considered as being statistically significant. Statistical analyses were performed using Statistical Package for Social Science $\left(\mathrm{SPSS}^{\circledR}\right.$, version 12.0) for Windows $\left(\mathrm{SPSS}^{\circledR}\right.$, Chicago, Illinois, USA).

\section{RESULTS}

Of 251 patients treated for diffuse secondary peritonitis $114(45.4 \%)$ were defined as PP and 137 (54.6\%) as CAP. The median daily evaluated APACHE II-score for all patients was 21.0. Within the PP group the APACHE II-score was significantly higher (median 22.0) compared to in the CAP group (median 20.0) (p $=0.012$ ). The mortality rate was also higher within the PP group, but was not significantly different compared to that of the CAP patients $(p=0.06)$. The characteristics for both study groups are presented in Table 1. The underlying cause for secondary diffuse peritonitis regarding the anatomic origin is depicted in Figure 1 a.

Statistical analyses revealed no significant differences regarding mortality and anatomic origin of sec-

Table 1. Characteristics of the study population: Biographical data and characteristics for PP and CAP groups.

\begin{tabular}{lccc}
\hline & PP & CAP & p-value \\
\hline Patients (n) & 114 & 137 & n.s. \\
Age (median) & 59.2 years & 64.5 years & n.s. \\
Gender (female/male) & $56 / 58$ & $68 / 69$ & n.s \\
APACHE II-score & 22.0 (median) & 20.0 (median) & 0.012 \\
MPI-score & 30.0 (median) & 27.0 (median) & 0.001 \\
ICU (days) & 14.2 (median) & 9.1 (median) & 0.005 \\
In-hospital mortality & $47.4 \%$ & $35.8 \%$ & n.s. $(0.060)$ \\
Abdominal lavages & 4.5 (median) & 2.5 (median) & 0.009 \\
Multi organ failure & $14.9 \%$ & $4.2 \%$ & 0.032 \\
$\quad$ Circulation failure & $52.2 \%$ & $47.9 \%$ & n.s. \\
Renal failure & $14.9 \%$ & $9.9 \%$ & n.s. \\
Lung failure & $43.3 \%$ & $32.4 \%$ & n.s. \\
\hline
\end{tabular}

Statistical significance was considered as $\mathrm{p} \leq 0.05\left(\boldsymbol{\chi}^{2}\right.$-Test $)$ 

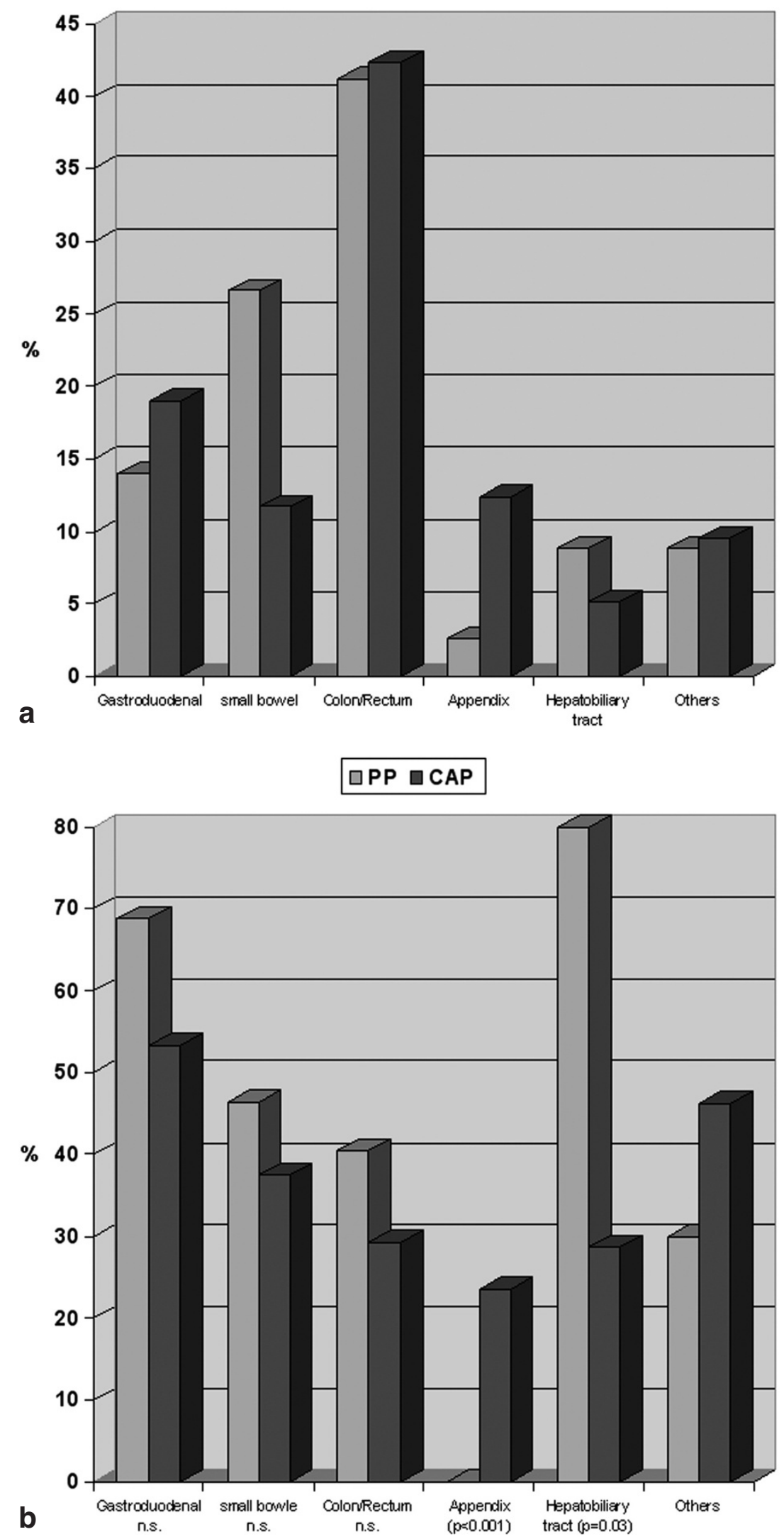

$\square P P \square C A P$

ondary peritonitis between the two groups except for the appendix $[4 / 17(\mathrm{CAP})$ vs. $0 / 3(\mathrm{PP}), \mathrm{p}=0.01]$ and the hepatobiliary tract $[2 / 7(\mathrm{CAP})$ vs. $8 / 10(\mathrm{PP}), \mathrm{p}=$ 0.03] (Fig. 1b).
Fig. 1.a Anatomical origin of secondary peritonitis. No statistically significant differences between PP and CAP ( $\mathrm{p}>0.05)$. $b$. Anatomical origin of secondary peritonitis and mortality.
A total of 93 patients $(81.6 \%)$ within the PP group and 133 patients $(97.1 \%)$ in the CAP group presented with abdominal pain at the time of physical examination $(\mathrm{p}=0.03)$. Abdominal rebound tenderness oc- 


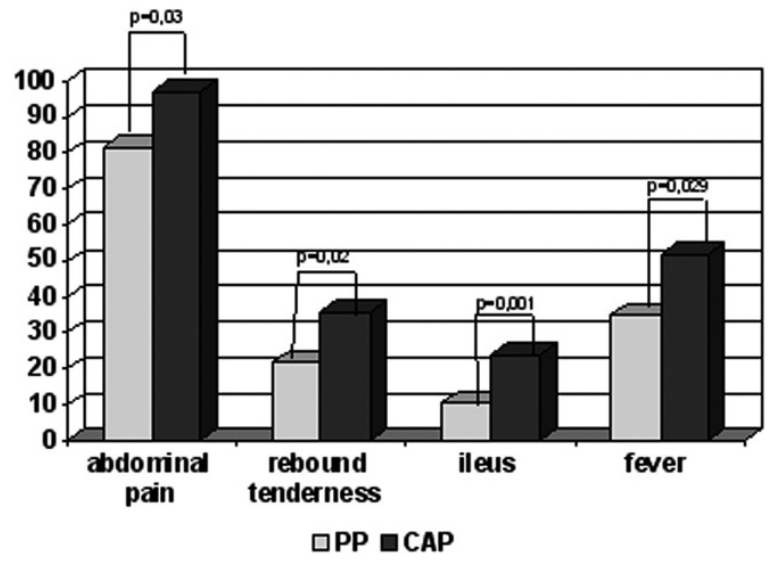

Fig. 2. Graphs depict the differences of clinical parameters. Clinical signs differ significantly between the two groups and are therefore of limited diagnotic value.

curred in 25 (PP, 21.9\%) and 49 (CAP, 35.8\%) patients, respectively $(\mathrm{p}=0.02)$. Furthermore, $40 \mathrm{pa}$ tients $(35.1 \%)$ within the PP group and 71 patients $(51.8 \%)$ within the CAP group, respectively, presented with fever exceeding $38.5^{\circ} \mathrm{C}(\mathrm{p}=0.029)$ (Fig. 2).

Over $95 \%$ of all patients presented with elevated infection parameters (WBC, CRP) but with no statistically significant difference between the two groups. Similarly, lactate and AT III values revealed no significant differences between the CAP and PP groups, respectively. Differences in clinical and paraclinical parameters for both groups are given in Table 2. PP was predicted correctly using diagnostic imaging in $97.2 \%$ (CT-scan), 66.2\% (Radiographs with contrast-medium) and $44.3 \%$ (Sonography), respectively (Table 3 ).
The causes for peritonitis in the PP group were as follows: Anastomotic leakage 50.9\% $(\mathrm{n}=58)$, perforations $22.8 \%(\mathrm{n}=26)$, mesenteric ischemia $11.4 \%(\mathrm{n}=$ 13) and others $14.9 \%(n=17)$.

The number of abdominal lavages was significantly higher in the PP group (4.5 median) compared to the CAP group (2.5 median) $(\mathrm{p}=0.009)$. In the CAP group one or two lavages were significantly more frequent than within the PP group (62.0\% vs. $40.7 \%$; p = $0.001)$. More than four lavages were performed more frequently in the PP group $(27.4 \%$ vs. $10.2 \%$; $<$ $0.001)$.

\section{Discussion}

Early detection and diagnosis in secondary peritonitis, either postoperative or community-acquired, is critical for defining the most effective treatment intervention. Clinical monitoring should be aimed at early identification of the source of the complication before secondary organ failure aggravates the clinical situation. The complication itself does not frequently represent the major problem, but is more likely a consequence of late diagnosis and therefore of insufficient therapy. Thus the identification of early predictive indicators of peritonitis and abdominal sepsis is of utmost importance $[10,11,13-16]$.

In our study we demonstrate that routinely used clinical and paraclinical parameters are of limited predictive value in the diagnosis of PP when compared to the group of patients with CAP. Slightly more than $50 \%$ of patients within the PP group presented with abdominal pain or tenderness to palpation accompanied by fever at the time of physical examination. Furthermore, clinical signs of peritonitis such as rigidity or

Table 2. Clinical and paraclinical parameters: Differences in clinical and paraclinical parameters of CAP and PP patients.

\begin{tabular}{lccc}
\hline & PP & CAP & p-value \\
\hline Abdominal pain & $93(81.6 \%)$ & $133(97.1 \%)$ & 0.03 \\
Abdominal rebound tenderness & $25(21.9 \%)$ & $49(35.8 \%)$ & 0.02 \\
Paralytic Ileus & $40(35.0 \%)$ & $33(24.1 \%)$ & n.s. \\
Fever $\left.>38.5^{\circ} \mathrm{C}\right)$ & $40(35.1 \%)$ & $71(51.8 \%)$ & 0.029 \\
WBC $[/ \mathrm{nl}]$ & 17.3 (median) & 18.0 (median) & n.s. \\
CRP $[\mathrm{mg} / \mathrm{l}]$ & 221.2 (median) & 205.1 (median) & n.s. \\
Lactate $[\mathrm{mmol} / \mathrm{l}]$ & 3.0 (median) & 3.4 (median) & n.s. \\
AT III $(\%)$ & 54.1 (median) & $59.6 \%$ (median) & 0.03 \\
Albumin $(\mathrm{g} / \mathrm{L})$ & 22.3 (median) & 25.7 (median) & 0.019 \\
Immunosuppression & $18(15.8 \%)$ & $8(5.8 \%)$ & 0.043 \\
MOF & $17(14.9 \%)$ & $6(4.2 \%)$ & \\
\hline
\end{tabular}

Table 3. Diagnostic significance in PP.

\begin{tabular}{lcccc}
\hline & Total & Correct & Incorrect & Sensitivity \\
\hline Sonography & 61 & 27 & 34 & $44.3 \%$ \\
Imaging with contrast medium & 77 & 51 & 26 & $66.2 \%$ \\
Mesenteric angiography & 6 & 35 & 1 & $50.0 \%$ \\
CT-scan & 36 & 35 & $97.2 \%$ & \\
\hline
\end{tabular}

Only CT-scan showed high sensitivity in diagnosing PP. 
rebound tenderness were only evident in $21.9 \%$ of these patients. This could be explained by the predominant post-aggression catabolism as well as by the postoperative administration of analgetic drugs [17]. These patients are therefore frequently diagnosed after signs of sepsis have already occurred [5, 7, 10, 15, 17-19].

The low accuracy of physical tests in diagnosing postoperative peritonitis reflects the questionable value of a so-called 'experienced surgeon'. Of course, clinical judgment remains important but seems to frequently fail in times of effective postoperative analgesia and sedation of intubated patients $[15,19]$. This is underlined by the fact that physical tests are not included in the 'top-ten ranked' variables for inpatient mortality of relaparotomy outcome in peritonitis patients [13]. The current situation is thus that physical tests do not yield meaningful variables with respect to prediction of ongoing abdominal sepsis.

Analysis of laboratory infection parameters such as leukocytosis and C-reactive protein revealed no significant difference between the two groups. Furthermore, levels of AT III and lactate as parameters for mesenteric ischemia and coagulopathy within the course of sepsis were also not significantly different. Other diagnostic parameters such as interleukin 6 and tumor necrosis factor $\alpha$ (TNF $\alpha$ ) levels are implicated as being important in the early onset of sepsis but do not correlate with outcome [20-22]. Moreover, their short half-lives make their diagnostic applicability questionable [23].

There has been great interest in the diagnostic and prognostic potential of procalcitonin in abdominal sepsis. Even though existing data support the sensitivity and specificity as an early marker for sepsis, its value is discussed controversial [8, 24, 25]. Recently, determination of procalcitonin levels in peritonitis was associated with a low sensitivity and specificity [26]. In severe abdominal inflammation such as peritonitis, other sources such as catheter infections, pulmonary or urinary tract infections have to be taken into account in critically ill patients.

Once secondary peritonitis is suspected clinically, further investigations such as abdominal ultrasound and radiograph-based imaging techniques are necessary to confirm the presence and source of peritonitis. In our study the use of abdominal ultrasonography only revealed a sensitivity of $46 \%$, possibly due to commonly occurring paralysis and/or meteorism of the intestines. Undoubtedly, mesentericography and radiograph imaging using contrast dyes have their value. Within this study, CT-scan revealed a sensitivity of $97.2 \%$ in detecting formation and source of peritonitis and was therefore the most precise and valuable imaging technique. Other studies support the utilization of CT-scanning in the diagnosis of secondary PP [27, 28].

An optimal scoring system should fulfill the following criteria: Objective, readily measurable under routine conditions, simple, easy available but also reliable, specific for the function of the organ considered and independent on the type of patient and therapeutic intervention. With the exception of the abdominal CTscan, the investigated clinical and laboratory parameters unfortunately did not meet these criteria [13]. Due to the retrospective character of the study only sensitivity and not specificity can be presented.
The mortality rate for patients within the PP group was $47.4 \%$ and therefore slightly higher than that for patients with CAP $(35.8 \%, \mathrm{p}=0.060)$. The current literature reports mortality rates between 25 and $68 \%$ for secondary peritonitis, whereas the rates for the subgroup of PP unexceptionally exceed those for CAP $[3-5,11,29]$. Even though there is a trend towards higher mortality in the PP group, rates did not differ significantly from the CAP group, which is a novel finding. Thus our results do not support an observation strategy in uncertain cases of peritonits without pathological findings on CT-scan as it has been advocated by others [14, 30]

The lack of statistical difference in mortality rates between the two groups are probably caused by the most early intervention, even in doubtful cases. The significantly higher number of lavages necessary in the PP group indicates that these patients are either diagnosed too late (despite most early indication for relaparotomy) or are more susceptible to bacteraemia due to predominant immunosuppression. Nevertheless, the re-laparotomy strategy was not an outcome measure in this study. Thus, no clear recommendations can be given from our data.

There is no ideal control group for patients with diffuse PP to assess the value of diagnostic parameters. A comparison with uncomplicated recovering patients having undergone elective abdominal surgery would give apparently clear results but would not reflect the group of importance - postoperative patients in the ICU in critical condition with the question of whether to perform relaparotomy or not. We therefore consider the group of patients with diffuse CAP to be the most appropriate due to the comparable extent of peritonitis and the severity of sepsis signs. The separation between diffuse CAP and PP is undoubtfully artificial. It might be more appropriate to compare patients with surgically proven peritonitis and patients suspicious for peritonitis but without surgical findings at the time of laparotomy. Due to the retrospective character of this study it is almost impossible to re-evaluate the latter group. Evaluating on the other hand - this more "ideal" study collective in a randomized controlled setting might increase the clinical awareness and therefore leading to an evaluation bias. Nevertheless, earlier diagnosis and consecutive treatment in these patients is frequently performed because of the more frequently apparent clinical symptoms. This has to be taken into account critically for the assessment of the results revealed by this study.

\section{CONCLUSION}

Despite advances in intensive care support, scoring systems and sophisticated markers, early diagnosis of generalized PP remains difficult. In our study, clinical and laboratory parameters were of limited value in the early diagnosis of PP. The most reliable imaging technique was the CT-scan. Only interventions prior to sepsis onset and development of MOF can reduce morbidity and mortality. We thus favour early relaparotomy even in suspected cases of PP as being the appropriate timepoint for intervention. 
Acknowledgments: Prof. Robert Harris, Karolinska Institutet, Stockholm is greatefully acknowledged for critical reviewing the manuscript and his helpful comments.

\section{REFERENCES}

1. Kujath P, Rodloff A: Peritonitis. Second edition, page 3641. ed Second edition Bremen, London, Boston, UNIMED, 2005.

2. Buchler MW, Baer HU, Brugger LE, Feodorovici MA, Uhl W, Seiler C: [Surgical therapy of diffuse peritonitis: debridement and intraoperative extensive lavage]. Chirurg 1997;68:811-815.

3. Pacelli F, Doglietto GB, Alfieri S, Piccioni E, Sgadari A, Gui D, Crucitti F: Prognosis in intra-abdominal infections. Multivariate analysis on 604 patients. Arch Surg 1996;131:641-645.

4. Pusajo JF, Bumaschny E, Doglio GR, Cherjovsky MR, Lipinszki AI, Hernandez MS, Egurrola MA: Postoperative intra-abdominal sepsis requiring reoperation. Value of a predictive index. Arch Surg 1993;128:218-222; discussion 223.

5. Anderson ID, Fearon KC, Grant IS: Laparotomy for abdominal sepsis in the critically ill. Br J Surg 1996;83:535539.

6. Kirschner: Die Behandlung der akuten eitrigen freien Bauchfellentzündung. Arch klin Chir 1926;142:253-311.

7. McLauchlan GJ, Anderson ID, Grant IS, Fearon KC: Outcome of patients with abdominal sepsis treated in an intensive care unit. Br J Surg 1995;82:524-529.

8. Rau BM, Frigerio I, Buchler MW, Wegscheider K, Bassi C, Puolakkainen PA, Beger HG, Schilling MK: Evaluation of procalcitonin for predicting septic multiorgan failure and overall prognosis in secondary peritonitis: a prospective, international multicenter study. Arch Surg 2007;142:134-142.

9. Unalp HR, Kamer E, Kar H, Bal A, Peskersoy M, Ali Onal M: Urgent abdominal re-explorations. World J Emerg Surg 2006;1:10.

10. van Ruler O, Lamme B, Gouma DJ, Reitsma JB, Boermeester MA: Variables associated with positive findings at relaparotomy in patients with secondary peritonitis. Crit Care Med 2007;35:468-476.

11. Lamme B, Boermeester MA, Belt EJ, van Till JW, Gouma DJ, Obertop H: Mortality and morbidity of planned relaparotomy versus relaparotomy on demand for secondary peritonitis. Br J Surg 2004;91:1046-1054.

12. Edelsberg J, Berger A, Schell S, Mallick R, Kuznik A, Oster $G$ : Economic consequences of failure of initial antibiotic therapy in hospitalized adults with complicated intraabdominal infections. Surg Infect (Larchmt) 2008;9:335347.

13. Lamme B, Mahler CW, van Ruler O, Gouma DJ, Reitsma JB, Boermeester MA: Clinical predictors of ongoing infection in secondary peritonitis: systematic review. World J Surg 2006;30:2170-2181.

14. Marshall JC, Innes M: Intensive care unit management of intra-abdominal infection. Crit Care Med 2003;31:22282237.

15. Mulier S, Penninckx F, Verwaest C, Filez L, Aerts R, Fieuws S, Lauwers P: Factors affecting mortality in generalized postoperative peritonitis: multivariate analysis in 96 patients. World J Surg 2003;27:379-384.

16. van Ruler O, Mahler CW, Boer KR, Reuland EA, Gooszen HG, Opmeer BC, de Graaf PW, Lamme B, Gerhards MF, Steller EP, van Till JW, de Borgie CJ, Gouma DJ, Reitsma JB, Boermeester MA: Comparison of on-demand vs planned relaparotomy strategy in patients with severe peritonitis: a randomized trial. Jama 2007;298:865-872.
17. Makela J, Kairaluoma MI: Relaparotomy for postoperative intra-abdominal sepsis in jaundiced patients. Br J Surg 1988;75:1157-1159.

18. Hinsdale JG, Jaffe BM: Re-operation for intra-abdominal sepsis. Indications and results in modern critical care setting. Ann Surg 1984;199:31-36.

19. Holzheimer RG, Gathof B: Re-operation for complicated secondary peritonitis - how to identify patients at risk for persistent sepsis. Eur J Med Res 2003;8:125-134.

20. Fang XM, Schroder S, Hoeft A, Stuber F: Comparison of two polymorphisms of the interleukin-1 gene family: interleukin-1 receptor antagonist polymorphism contributes to susceptibility to severe sepsis. Crit Care Med 1999;27:1330-1334.

21. Harbarth S, Holeckova K, Froidevaux C, Pittet D, Ricou B, Grau GE, Vadas L, Pugin J: Diagnostic value of procalcitonin, interleukin-6, and interleukin-8 in critically ill patients admitted with suspected sepsis. Am J Respir Crit Care Med 2001;164:396-402.

22. Majetschak M, Flohe S, Obertacke U, Schroder J, Staubach K, Nast-Kolb D, Schade FU, Stuber F: Relation of a TNF gene polymorphism to severe sepsis in trauma patients. Ann Surg 1999;230:207-214.

23. Reith HB, Mittelkotter U, Debus ES, Kussner C, Thiede A: Procalcitonin in early detection of postoperative complications. Dig Surg 1998;15:260-265.

24. Reith H, Mittelkötter U, Wagner R, Thiede A: Procalcitonin (PCT) in patients with abdominal sepsis. Intensive Care Med 2000;26(suppl2):159-164.

25. Schroder J, Staubach KH, Zabel P, Stuber F, Kremer B: Procalcitonin as a marker of severity in septic shock. Langenbecks Arch Surg 1999;384:33-38.

26. Lam MF, Leung JC, Lam CW, Tse KC, Lo WK, Lui SL, Chan TM, Tam S, Lai KN: Procalcitonin fails to differentiate inflammatory status or predict long-term outcomes in peritoneal dialysis-associated peritonitis. Perit Dial Int 2008;28:377-384.

27. Evans HL, Raymond DP, Pelletier SJ, Crabtree TD, Pruett TL, Sawyer RG: Diagnosis of intra-abdominal infection in the critically ill patient. Curr Opin Crit Care 2001;7:117-121.

28. Rotstein OD, Meakins JL: Diagnostic and therapeutic challenges of intrababominal infections. World J Surg 1990;14:159-166.

29. Nathens AB, Rotstein OD, Marshall JC: Tertiary peritonitis: clinical features of a complex nosocomial infection. World J Surg 1998;22:158-163.

30. Marshall JC, Vincent JL, Fink MP, Cook DJ, Rubenfeld G, Foster D, Fisher CJ, Jr., Faist E, Reinhart K: Measures, markers, and mediators: toward a staging system for clinical sepsis. A report of the Fifth Toronto Sepsis Roundtable, Toronto, Ontario, Canada, October 25-26, 2000. Crit Care Med 2003;31:1560-1567.

Received: May 2, 2009 / Accepted: June 9, 2009

Address for correspondence:

Dr. med. Franz G. Bader, MD, PhD

Department of Surgery

University of Schleswig-Holstein

Campus Lübeck

Ratzeburger Allee 160

23538 Lübeck

Germany

Tel.: $\quad++49(0) 451 / 5002001$

Fax: $\quad++49(0) 451 / 5002069$

E-mail: franz_bader@yahoo.de 